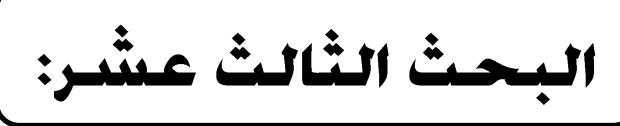

Lifestyle Matters: The Effect of Lifestyle-Behaviors and SelfPerceived Health on Psychological Resilience during COVID-19 Pandemic

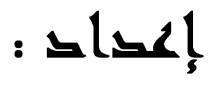

ImanAlyAlmohammadi, PhD

Department of Psychology, Faculty of Arts and Humanities, King AbdulAziz University Jeddah, KSA 



\title{
Lifestyle matters: the effect of Lifestyle-Behaviors and Self-perceived Health on Psychological Resilience during COVID-19 Pandemic
}

\section{ImanAlyAlmohammadi, PhD}

\author{
Department of Psychology, Faculty of Arts and Humanities, King AbdulAziz \\ University Jeddah, KSA \\ E-mail: ealmhamdi@kau.edu.sa
}

\begin{abstract}
This study investigates the effects of lifestyle-behaviors, self-perceived health, and income on resilience during COVID-19 pandemic in a sample of Saudi adults $(N=778)$. The Arabic version of the Connor-Davidson resilience scale was used. The results show that: individuals who report eating a balanced diet $(M=71.21)$ have higher resilience scores compared to participants who do not eat a balanced diet $(M=66.45), t(776)=4.51, p=$ .001; $d=0.33)$; individuals who exercise regularly have a higher level of resilience $(M=71.09)$ than individuals who do not exercise $(M=67.79)$, $t(776)=2.98, p=.001 ; d=0.23)$; there was no effect for good sleeping on resilience, $t(776)=.692, p=.489)$; participants who rate their health as very $\operatorname{good}(M=71.33)$ compared to individuals who rate their health as good $(M=$ 65.39) demonstrated better resilience scores, $t(760)=5.50, p=.001 ; d=$ $0.41)$; and there was a statistically significant differences between income groups $(F(3,774)=13.24, p=.001 ; \eta 2=0.05)$. It was recommended that interventions to promote a healthy lifestyle should be undertaken to improve the psychological resilience level of individuals during the COVID-19 pandemic.
\end{abstract}

Keywords:Psychological Resilience; Lifestyle-behaviors; Exercise; Diet, COVID-19.

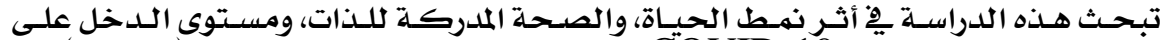

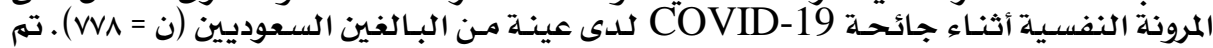

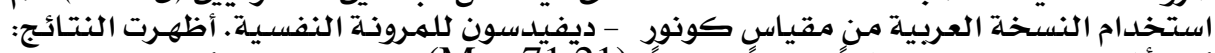

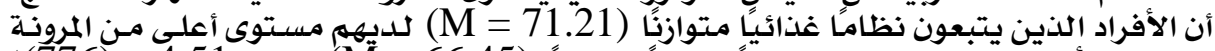

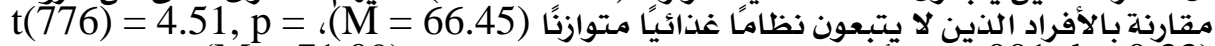

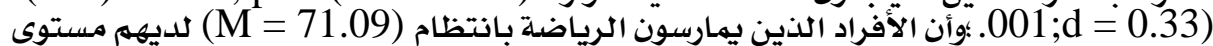

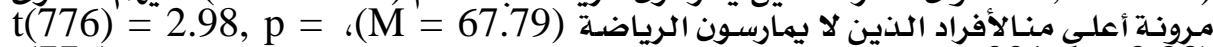

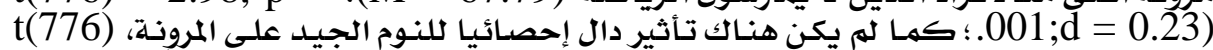

(M = أ.692, p = =.489)

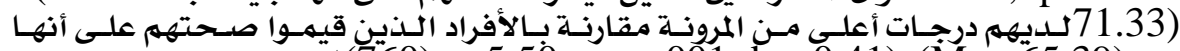

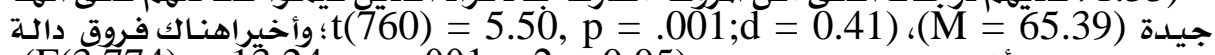

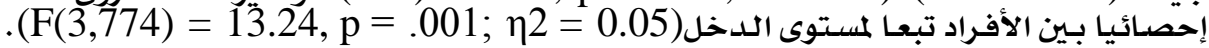

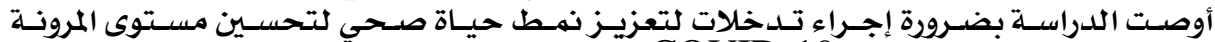

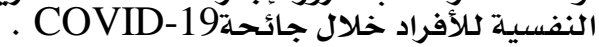
الكلمات المفتاحية: المروخة النفسية؛ نمط الحياة ؛التمارين الرياضية؛ النظام الغذائي؛ كوفيد 


\section{Introduction}

The coronavirus (COVID-19) outbreak is the greatest challenge facing our world today. It starts in China late 2019, and since, the virus has spread almost to every continent. It is the biggest global public health crisis in our time due to several reasons: (1)the speed of the virus spread, the number of new cases increases constantly each day; there are currently 203,295,170 confirmed cases and 4,303,515 deaths from the coronavirus COVID-19 outbreak as of August11, 2021 (WHO, 2020a). (2) It transmits easily from person to person; experts believe that the virus spreads in several ways, e.g.droplets or aerosols from coughing, sneezing, or talking,also, it can transmit via air and surface; the virus can live up to 3 hours in the air and up to 2 to 3 days on surfaces like plastic and stainless steel (WEBMED, 2020). (3) It is a new virus; according to the World Health Organization (WHO), there were no specific vaccines or treatments for COVID-19 when this study was conducted between May - June 2020 (WHO, 2020b). Therefore, COVID-19 has been characterized as a pandemic by the World Health Organization on March 11 2020 (WHO, 2020c).

However, the pandemic is not just a public health crisis, but also an unprecedented socio-economic crisis that severely affected the globe. Pak et al. (2020) pointed out the consequences of the measures that have been implemented in many countries to slow down the spread of the Coronavirus; there is a significant decrease in income, anincrease in unemployment, and disruptions in the transportation, service, and manufacturing industries.

Undoubtedly,this disruption in our lifestyle, daily routine, business, and even the education system, in addition, the unpredictability and uncertaintyaround the coronavirus outbreak, can create an atmosphere that triggers stress, anxiety, fear, and sometimes panic.A growing body of evidence from recent studies has shown the negative psychological impact of COVID-19 on the public (El-Hage et al.,2020; Dubey et ai., 2020; Gualano et al., 2020; Khan et al., 2020; Mukhtar, 2020; Neto et al., 2020;Zhang \& Ma, 2020). For instance, Wang et al. (2020) conducted a study on 1210 individuals from 194 cities in China, their results show that in the early stage of

\section{$\varepsilon \varepsilon 7$}


the COVID-19 outbreak in China, more than half of the participants rated the psychological impact as moderate-to-severe, and about onethird reported moderate-to-severe anxiety. Li et al. conducted two studies (2020-a; 2020b) on 4607 Chinese; the first study shows that persons' cognitive appraisals, perceived severity of Coronavirus, are related to severalunwanted emotional and behavioral reactions like an increase in negative emotion and sleep problems. The second study reveals that perceived severity of COVID-19 was positively related to mental health problems. Another study (Ying et al., 2020) was conducted among 854 family members of health care workers in designated hospitals in Ningbo, China, during the COVID-19 epidemic. The results show a high prevalence of anxiety and depression symptoms among the participants.

Understandably, people respond differently to stressful situations like COVID-19 pandemic; some seem to cope better and deal well with the situation while others have strong and lingering reactions like disbelief, feeling sad or angry, frustrated, and helpless. In general, People's reaction to the crisis dependson several factors such as physical and mental health status, financial state, family support, and awareness level (Hsu et al., 2020).

Prior literature suggests the important role of psychological resilience in hard times and traumatic events such as COVID-19 pandemic (Nugent et al., 2014;Peres et al, 2007; Thompson et al, 2011). Psychological resilienceis described as a "rebound ability" that allows humans to maintain good adaptability in the face of adversity, trauma, tragedy, threats, or significant sources of stresssuch as family and relationship problems, serious health problems, or workplace and financial stressors (APA, 2012).Vinkers et al. (2020) stated that psychological resilience is essential to cope with stress and important to stay in balance during the coronavirus pandemic.Another study, conducted on 600 medical staff members from 32 public hospitals in China(Huang et al., 2020), showed that there was a significant negative correlation between perceived stress and psychological resilience. The authors recommended that interventions should be undertaken to improve the psychological resilience level of the medical staff during the outbreak of COVID-19. Similarly, 


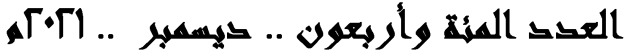

Khanmohammadi et al. (2020) found a significant negative correlation between psychological resilience and occupational stress among Iranian nurses during COVID-19 pandemic. Also, a recent study conducted in Turkey on 518 participants (Karaşar\&Canli, 2020), the results showed a significant negative correlation between psychological resilience and depression.

It has been proven that psychological resilience is the tool that enable people to cope and even thrive in time of stress and hardship. However, psychological resilience level can be affected by our lifestyle and daily routine(Gilles, n.d; NZDF, n.d.) like diet (Bonaccio et al., 2017; Lutz et al., 2016; Whatnall et al., 2019), exercise (Childs \& de Wit, 2014; Chow \& Choi, 2019;Deuster\& Silverman, 2013; Yu et al., 2020), sleep (Blanc et al., 2020; Grossman et al., 2021; Hughes et al., 2018; Wang et al., 2020). Furthermore, psychological resilience can be influenced by self-perceived health (Lynskey et al., 2020; Schure et al.,2013; Wells et al., 2012) and income (Ferguson, Bender, \& Thompson, 2018;Mizrak\&Tutkun, 2020).

SinceCoronavirus presents a threat to our health, economy, and social unity of societies on a global level, there is an urgent need to center our attention on psychological resilience.Therefore, the aim of this study is to examine the effects of lifestyle-behaviors such as eating a balanced diet, exercise, and good sleep, as well asself-perceived health and monthly income on our psychological resilience during COVID-19 pandemic. It was hypothesized that:(1) H1: Participants who eat a balanced diet will report higher levels of psychological resilience than those who do not eat a balanced diet ; (2) $\mathrm{H}_{2}$ : participants who exercise regularly will report higher levels of psychological resilience than those who do not exercise regularly; (3) $\mathrm{H}_{3}$ : participants who report having a good sleep every night will have higher levels of psychological resilience than those who do not report having a good sleep every night; $(4) \mathrm{H}_{4}$ :participants who rate their health as very good will report higher levels of psychological resilience than those who rate their health as good; (5)H5: the higher the income for participants, the more they will likely report higher levels of psychological resilience. 


\section{Method}

\section{Design}

A causal-comparative study design (Mertler, 2011).

\section{Participants}

Participants were population-based Saudi adults $(\mathrm{N}=778)$, who were recruited online via emails and social media from across the Kingdom of Saudi Arabia from May 2020 to June 2020. Seventy four percent of participants were females. Mean age was 32.11, ranging from 18 to 74 years. Table 1 displays a complete listing of the demographic variables of the sample.

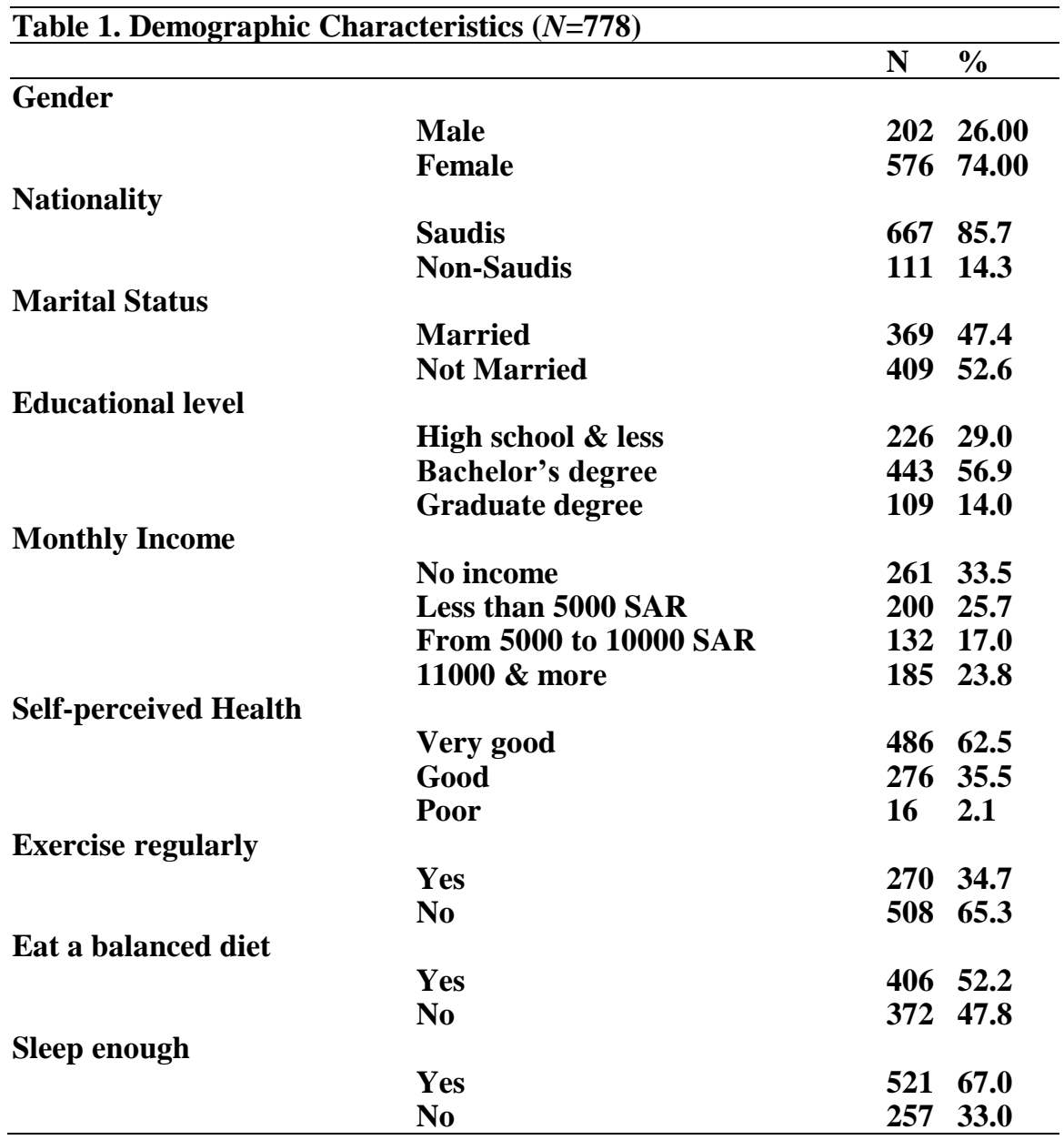




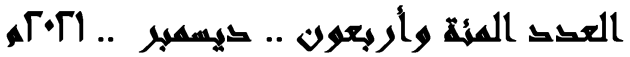

\section{Measures}

A demographic questionnaire, lifestyle behaviors questionnaire, and the Connor-Davidson Psychological resilience scale (CD-RISC) were included in this study.

Demographic Questionnaire. Questions about participant's age, gender, education level, income, Marital status, Self-perceived healthstatus.

Lifestyle Behaviors Questionnaire. Consists of three questions: (1) Do you exercise regularly, at least 150 minutes of moderate-intensity activity, or 75 minutes of vigorous-intensity activity every week, or a combination of the two together? (2) In general, is your diet healthy and balanced, includes carbohydrates, proteins, fats, vitamins, and minerals? (3) in general, do you get enough sleep (from 7-9 hours each night)?

The Connor-Davidson Psychological resilience scale (CDRISC).The CD-RISC consists of twenty-five items rated on a 5-point scale (0-4), with higher scores reflecting increased psychological resilience. It has existing evidence of reliability $(\alpha=.89)$ (Connor and Davidson, 2003). The CD-RISC has five distinct factors: personal competence, high standards, and tenacity; trust in one's instincts, tolerance of negative affect, and strengthening effects of stress; the positive acceptance of change and secure relationships;control; and spiritual influences (Connor and Davidson, 2003).

\section{Statistical Analysis}

All the following analyses were performed using the IBM Statistical Package for Social Sciences (SPSS) version 27.0.

- Descriptive statistics: frequencies, percentages, means, and standard deviations.

- The Cronbach's coefficient Alpha (Cronbach, 1951) to assess the CD-RISC reliability.

- Analysis of variance (ANOVA) or independent sample $t$ testto compare the means among the participants groups.

- Cohen's d and Eta squared $\eta^{2}$ effect size estimates.

\section{Results}




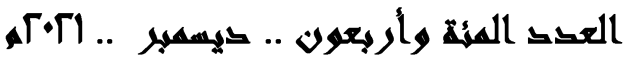

$H_{1}$ : Participants who eat a balanced diet will report higher levels of psychological resilience than those who do not eat a balanced diet.

Results of $t$ tests indicated that individuals who report eating a balanced $\operatorname{diet}(M=71.21, S D=13.56)$ have significantly higher psychological resilience score compared to participants who do not eat a balanced $\operatorname{diet}(M=66.45, S D=15.65), t(776)=4.51, p=.001 ; d=0.33)$.

$\mathrm{H}_{2}$ : Participants who exercise regularly will report higher levels of psychological resilience than those who do not exercise regularly.

Results of $t$ tests showed that individuals who exercise regularly have a higher level of psychological resilience $(M=71.09, S D=14.32)$ than individuals who do not exercise regularly $(M=67.79, S D=15.90)$, $t(776)=2.98, p=.001 ; d=0.23)$.

$H_{3}$ : Participants who report having a good sleep every night will have higher levels of psychological resilience than those who do not report having a good sleep every night.

Results of $t$ tests revealed the absence of significant differences, $t(776)$ $=.692, \mathrm{p}>.05)$ between Participants who report having a good sleep every night $(\mathrm{M}=69.19, \mathrm{SD}=14.72)$ andparticipants who do not report having a good sleep every night $(\mathrm{M}=68.41, \mathrm{SD}=14.91)$.

$\mathrm{H}_{4}$ : Participants who rate their health as very good will report higher levels of psychological resilience than those who rate their health as good.

Results of $t$ tests indicated that participants who rate their health as very good $(M=71.33, S D=14.01)$ compared to individuals who rate their health as good $(M=65.39, S D=14.88)$ demonstrated significantly better psychological resilience score, $t(760)=5.50, p=.001 ; d=0.41$ ).

$\mathrm{H}_{5}$ : The higher the income for participants, the more they will likely report higher levels of psychologicalresilience.

The results suggested that there was a statistically significant differences between income groups as determined by one-way ANOVA $\left(F(3,774)=13.24, p=.001 ; \eta^{2}=0.05\right)$. Post hoc analyses using theTukey test for significance indicated that the psychological resilience mean of participants with income from 5000 to 10000 $\operatorname{SAR}(M=72.14, \mathrm{SD}=14.67)$ was significantly higher $(\mathrm{p}=.001)$ than the other two groups: the participant with income Less than 5000 SAR $(\mathrm{M}=67.22, \mathrm{SD}=14.45)$ and the participants with no income $(\mathrm{M}=$ 
$65.59, \mathrm{SD}=14.99$ ). Moreover, the results showed that psychological resilience mean of the participants with income of 11000 SAR and more $(\mathrm{M}=73.23, \mathrm{SD}=13.44)$ was significantly higher $(\mathrm{p}=.001)$ than the other two groups: the participant with income Less than 5000 SAR $(\mathrm{M}=67.22, \mathrm{SD}=14.45)$ and the participants with no income $(\mathrm{M}=$ 65.59 , SD = 14.99). Finally, there were no statistically significant differences between the participants with no income and the participant with income less than 5000 SAR ( $p>.05)$, as well as, between the participants with income from 5000 to 10000 SAR and the participants with income of 11000 SAR and more $(p>.05)$.

\section{Discussion}

This study was conducted after the World Health Organization declared COVID-19 as a pandemic on March 11th, 2020, and during the Mandatory home quarantine in Saudi Arabia. The purpose of this study was to examine the effects of lifestyle-behaviors such as eating a balanced diet, exercise, and good sleep, as well as self-perceived health and monthly income on our psychological resilience during COVID-19 pandemic.

The results demonstrated that participants who eat a balanced diet reported higher levels of psychological resilience than those who do not eat a balanced diet (H1).This result could be considered in line with the findings emerged by a study carried out by Lutz and colleagues (2016) to assess the relationship between diet quality and psychological psychological resilience with 834 participants from the Army and Air Force; their results showed that better diet quality was associated with psychological resilience; they concluded that small improvements in diet quality canbe associated with better psychological resilience levels.Likewise, Bonaccio et al. (2017) found that Mediterranean diet, vegetable-based dietary, and better diet quality in general were all positivelylinkedto higher psychological resilience levels among 10812 subjects from a general adult population. There is also consistency between this finding witha studyconducted in a sample of 2710 Australianuniversity students (Whatnall et al., 2019), the researchers found that higher psychological resilience was correlated with higher 
fruit and vegetable serves a day, more regular breakfast consumption, and less frequent soft drink and takeaway food consumption.

The findingsalso corroborated that participants who exercise regularly reported higher levels of psychological resilience than those who do not exercise regularly $(\mathrm{H} 2)$. Thisresultis consistent with previousresearch, as Deuster\& Silverman (2013) presented arguments to emphasize the role of Physical fitness as a pathway to psychological resilience. They argued that physical fitness grants physiological and psychological benefits, reduces stress reactivity, serves as a shield against stress, and can protect against stress-related disorders and chronic illness. Similarly, Childs \& de Wit (2014) found that respondents who exercise regularly showed less decrease in positive affect after stress than sedentary respondents among 111 healthy individuals. Their findings support that habitual exercise is associated with stress psychological resilience in healthy individuals. Also, Chow \& Choi (2019) found low positive correlation between psychological resilience and physical activity.Anotherrecent study by (Yu et al., 2020) revealed that psychological resilience was positively correlated with occupational physical activity, moderate to vigorousphysical activity at work and dynamic standing at work in a sample of 93 nurses from four intensive care units in New Zealand.

However, the results disconfirmed that participants who report having a good sleep every night had higher levels of psychological resilience than those who do not report having a good sleep every night (H3).This outcome is contrary to previous studies, for example, Wang et al. (2020) found that higher psychological resilience scores predicted overall better sleep quality and shorter sleep latency in a sample of 1299 students, but not vice versa. Their results suggest that sleep problems may have more far-reaching effects on psychological resilience. Another recent study (Grossman et al., 2021) indicated that sleep problems correlated negatively with psychological resilience in 243 older adults. Likewise, Blanc et al. (2020) found that higher psychological resilience factors were strongly associated with fewer sleep disturbance symptoms among 700 older females. Also, Hughes et al. (2018) found that poor sleeping Veterans had lower psychological resilience. 


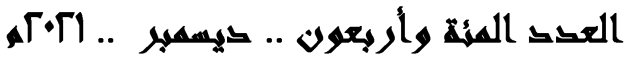

The present study also found thatparticipants who rate their health as very good reported higher levels of psychological resilience than those who rate their health as good (H4).This finding is in line with a previous study of Lynskey et al. (2020) who found a strong positive correlation between psychological resilience and reported health status in 140 hip and knee arthroplasty patients. Similarly, Wells et al. (2012) reported that older women, who happened to be Roman Catholic nuns in their study, with better health had higher psychological resilience levels. Besides Schure et al. (2013) confirm the association between health and psychological resilience. Their results showed that higher levels of psychological resilience were associated with lower levels of depressive symptomatology chronic pain, and with higher levels of mental and physical healthamong older American Indians.

Similarly, the results confirmed that the higher the income for participants, the more they were likely to report higher levels of psychological resilience (H5). This result seems to be in agreement with those obtained byFerguson, Bender, \& Thompson (2018) who studied 601 homeless young adults from three U.S. cities. They found that income generation from formal sources was associated with reporting higher levels of psychological resilience. Further support of this finding found in a study conducted in Turkey by Mizrak\&Tutkun (2020). They reported that the most resilient subjects are those with a monthly income of more than 3001 TL.

The observed results should be interpreted in light of limitations. One limitation of thepresent study is that this was a cross-sectional study; future research is needed to examine the link between these independent variables and psychological resilience over time. Another limitation, a population-based sample was used in this study; the findings should be examined indifferent samples; like adolescents, college students, or individuals with illnesses related to lifestylebehaviors (e.g. heart diseases, pre-diabetes or diabetes, and obesity).

\section{Conclusion and recommendation}

To conclude, this is one of the first studies that assess psychological resilience in relation to lifestyle-behaviors, self-perceived health, and monthly income during COVID-19 pandemic in Saudi 


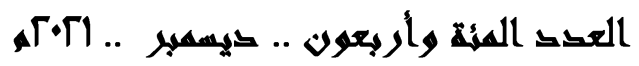

adults population. The results highlight a potential link between lifestyle-behaviors and psychological resilience, therefore, it is recommended that future interventions and awareness campaigns, that intended to increase people psychological resilience in difficult time such as COVID-19 pandemic, should promote for healthy lifestyle which combineseating a balanced diet, exercise, and enough sleep. Of course, further research in this area is warranted.

\section{References}

- American Psychological Association (2012). Building your resilience. Retrieved August 24, 2020, from https://www.apa.org/topics/ psychological resilience

- Blanc, J., Seixas, A., Donley, T., Bubu, O. M., Williams, N., \& JeanLouis, G. (2020). resiliencefactors, race/ethnicity and sleep disturbance among diverse older females with hypertension. Journal of affective disorders, 271, 255-261. https://doi.org/10.1016/j.jad. 2020.03.148

- Bonaccio, M., Di Castelnuovo, A., Costanzo, S., Pounis, G., Persichillo, M., Cerletti, C..\&Iacoviello, L. (2018). Mediterraneantype diet is associated with higher psychological resilience in a general adult population: findings from the Moli-sani study. European journal of clinical nutrition, 72(1), 154-160. https://doi.org/10.1038/ ejcn.2017.150

- Childs, E., \& de Wit, H. (2014). Regular exercise is associated with emotional resilience to acute stress in healthy adults. Frontiers in physiology, 5, 161. https://doi.org/10.3389/fphys.2014.00161

- Chow, S. \& Choi, E. (2019). Assessing the Mental Health, Physical Activity Levels, and resilienceof Today's Junior College Students in Self-Financing Institutions. International journal of environmental research and public health, 16(17), 3210. https://doi.org/10.3390/ ijerph16173210

- Connor KM and Davidson JRT (2003) Development of a new resilience scale: The Connor-Davidson resilience Scale (CD-RISC). Depression and Anxiety 18(2): 76-82.

- Cronbach, L. J. (1951). Coefficient alpha and the internal structure of tests. Psychometrika, 16, (3), 297-334.

- Deuster, P. A., \& Silverman, M. N. (2013). Physical fitness: a pathway to health and resilience. U.S. Army Medical Department journal, 24-35. 
- Dubey, S., Biswas, P., Ghosh, R., Chatterjee, S., Dubey, M. J., Chatterjee, S., Lahiri, D., \&Lavie, C. J. (2020). Psychosocial impact of COVID-19. Diabetes \& metabolic syndrome, 14(5), 779-788. Advance online publication. https://doi.org/10.1016/j.dsx.2020.05.035 - El-Hage, W., Hingray, C., Lemogne, C., Yrondi, A., Brunault, P., Bienvenu, T., ....\&Aouizerate, B. (2020). Health professionals facing the coronavirus disease 2019 (COVID-19) pandemic: What are the mental health risks?]. L'Encephale, 46(3S), S73-S80. https://doi.org/ 10. 1016/j. encep.2020.04.008

- Ferguson, K., Bender, K., \& Thompson, S. (2018). Risk and resilience Factors Associated with Formal and Informal Income Generation Among Homeless Young Adults in Three U.S. Cities. Youth \& Society, 50(3), 351-376.https://doi.org/10.1177/0044118X15600722.

- Ghandehary, M., Shahri, M. \&Mohamadi, F. (2019). Mental health status and resilience of female prisoners with an interventional role of physical activity. Journal of Research \& Health,9 (6), p488-495. 8p.

- Gilles, G. (n.d.). Build up your resilience to Stressful Life Events. Retrieved August 25, 2020, from https://www.mentalhelp.net /blogs/build-up-your-psychological resilience -to-stressful-life-events/

- Grossman, E., Hoffman, Y., Palgi, Y., \&Shrira, A. (2021). COVID-19 related loneliness and sleep problems in older adults: Worries and resilience as potentialmoderators. Personality and individual differences, 168, 110371. https://doi.org/10.1016/j.paid.2020.110371

- Gualano, M., Lo Moro, G., Voglino, G., Bert, F. \&Siliquini, R. (2020). Effects of Covid-19 Lockdown on Mental Health and Sleep Disturbances in Italy. Int. J. Environ. Res. Public Health, 17, 4779.

- Hsu, C.-H., Lin, H.-H., Wang, C.-C., \&Jhang, S. (2020). How to Defend COVID-19 in Taiwan? Talk about People's Disease Awareness, Attitudes, Behaviors and the Impact of Physical and Mental Health. Int. J. Environ. Res. Public Health, 17, 4694.

- Huang, L., Wang, Y., Liu, J., Ye, P., Cheng, B., Xu, H., Qu, H., \& Ning, G. (2020). Factors Associated with resilience Among Medical Staff in Radiology Departments During the Outbreak of 2019 Novel Coronavirus Disease (COVID-19): A Cross-Sectional Study. Medical science monitor: international medical journal of experimental and clinical research, 26, e925669. https://doi.org/10.12659/MSM.925669 


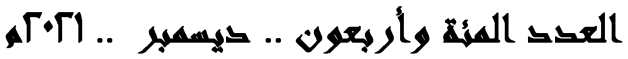

- Hughes, B.B., Lummis, S.C., Anderson, S.C., Kroeker, K.J., 2018. Unexpected resilience of a seagrass system exposed to global stressors. Glob. Chang. Biol. 24 (1), 224-234. https://doi.org/ $10.1111 / \mathrm{gcb} .13854$.

- Karaşar, B. \&Canli, D. (2020). Psychological resilience and Depression during the Covid-19 Pandemic in Turkey. Psychiatria Danubina,32 (2), pp. 273-279.

- Khan, S., Siddique, R., Li, H., Ali, A., Shereen, M. A., Bashir, N., \&Xue, M. (2020). Impact of coronavirus outbreak on psychological health. Journal of global health,10(1), 010331. https://doi.org/ 10.7189/jogh.10.010331

- Khanmohammadi, S., Hajibeglo, A., Rashidan, M. \&Bekmaz, K. (2020). Relationship of resilience with occupational stress among nurses in coronavirus ward of Khatam Al-Anbia Hospital, GonbadKavous, 2020. Neuropsychiatry \& Neuropsychology / Neuropsychiatria i Neuropsychologia, 15(1), 1-6. https://doi.org/ 10.5114/nan.2020.97397

- Li, J.-B., Yang, A., Dou, K., Wang, L.-X., Zhang, M.-C. \& Lin, X.-Q. (2020a). Chinese public's knowledge, perceived severity, and perceived controllability of the COVID-19 and their associations with emotional and behavioural reactions, social participation, and precautionary behaviour: A national survey. (Manuscript under review).

- $\underline{\text { Li}}$, J.-B., Yang, A., Dou, K. \& Cheung, R. (2020b). Self-Control Moderates the Association Between Perceived Severity of Coronavirus Disease 2019 (COVID-19) and Mental Health Problems Among the Chinese Public. International journal of environmental research and public health, 17, 4820; doi:10.3390/ijerph17134820

- Lutz, L., Gaffney-Stomberg, E., Williams, K., McGraw, S., Niro, P., Karl, J. .... \& McClung, J. (2016). Adherence to the Dietary Guidelines for Americans Is Associated with Psychological resilience in Young Adults: A Cross-Sectional Study. Journal of the Academy of Nutrition and Dietetics, 117(3), 396-403. https://doi.org/10.1016/ j.jand.2016.09.018

- Lynskey, S., Ling, F., Greenberg, A., Penny-Dimri, J., \& Sutherland, A. (2020). The influence of patient resilience and health status on satisfaction after total hip and knee arthroplasty. The surgeon: journal 


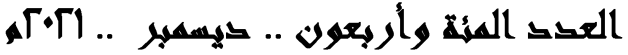

of the Royal Colleges of Surgeons of Edinburgh and Ireland, (19),814.

- Mertler, C. A., \& Charles, C. M. (2011). Introduction to educational research. Boston: Pearson/Allyn \& Bacon.

- Mizrak, S. \&Tutkun, T. (2020). Psychological resilience Level of Individuals Living in Çanakkale, Turkey . Educational Policy Analysis and Strategic Research, 15(1), 178-196. doi: 10.29329/epasr. 2020.236.10

- Mukhtar, S. (2020). Psychological health during the coronavirus disease 2019 pandemic outbreak. International Journal of Social Psychiatry, 66(5), 512-516. https://doi.org/10.1177/00207640209258 $\underline{35}$

- Neto, M., Almeida, H., Esmeraldo, J., Nobre, C., Pinheiro, W., de Oliveira, C., Sousa, I., Lima, O., Lima, N., Moreira, M. M., Lima, C., Júnior, J. G., \& da Silva, C. (2020). When health professionals look death in the eye: the mental health of professionals who deal daily with the 2019 coronavirus outbreak. Psychiatry research, 288, 112972. https://doi.org/10.1016/j.psychres.2020.112972

- New Zealand Defence Force. (n.d.). Everyday resilience: A practical guide to increasing performance. Retrieved August 25, 2020, from http://health.nzdf.mil.nz/assets/Uploads/Everyday-Psychological resilience .pdf

- Nugent, N. R., Sumner, J. A., \&Amstadter, A. B. (2014). resilience after trauma: from surviving to thriving. European journal of psychotraumatology, 5,10.3402/ejpt.v5.25339.https://doi.org/10.3402 / ejpt.v5.25339

- Pak, A., Adegboye, O., Adekunle, A., Rahman, K., McBryde, E. \& Eisen, D. (2020). Economic Consequences of the COVID-19 Outbreak: the Need for Epidemic Preparedness. Frontiers in Public Health, 8, 241. doi=10.3389/fpubh.2020.00241

- Peres, J., Moreira-Almeida, A., Nasello, A. \& Koenig, H. (2007). Spirituality and resilience in Trauma Victims. $J$ Relig Health 46, 343-350. https://doi.org/10.1007/s10943-006-9103-0

- Schure, M. B., Odden, M., \& Goins, R. T. (2013). The association of resilience with mental and physical health among older American Indians: the Native Elder Care Study. American Indian and Alaska native mental health research (Online), 20(2), 27-41. https://doi.org/10.5820/aian.2002.2013.27 


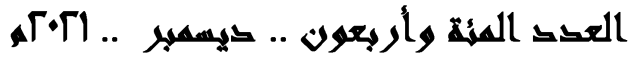

- Thompson, R., Arnkoff, D., \& Glass, C. (2011). Conceptualizing Mindfulness and Acceptance as Components of Psychological resilience to Trauma. Trauma, Violence, \& Abuse, 12(4), 220235. https://doi.org/10.1177/1524838011416375

- Vinkers, C., Amelsvoort, T., Bisson, J., Branchi, I., Cryan, J., Domschke, K. ....Wee, N. (2020). Stress resilience during the coronavirus pandemic. European Neuropsychopharmacology,35, PP.12-16. https://doi.org/10.1016/j.euroneuro.2020.05.003

- Wang, C., Pan, R., Wan, X., Tan, Y., Xu, L., Ho, C., \& Ho, R. (2020). Immediate Psychological Responses and Associated Factors during the Initial Stage of the 2019 Coronavirus Disease (COVID-19) Epidemic among the General Population in China. International journal of environmental research and public health, 17(5), 1729. https://doi.org/10.3390/ijerph17051729

- Wang, J., Zhang, X., Simons, S. R., Sun, J., Shao, D., \& Cao, F. (2020). Exploring the bi-directional relationship between sleep and resilience in adolescence. Sleep medicine, 73, 63-69. https://doi.org/ 10.1016/i.sleep.2020.04.018

- WebMD Medical Reference (2020, August). How Does Coronavirus Spread? Retrieved August 11, 2020, from https://www.webmd.com/ lung/coronavirus-transmission-overview\#1

- Wells, M., Avers, D., \& Brooks, G. (2012). resilience, physical performance measures, and self-perceived physical and mental health in older Catholic nuns. Journal of geriatric physical therapy, 35(3), 126-131. https://doi.org/10.1519/JPT.0b013e318237103f.

- Whatnall, M. C., Patterson, A. J., Siew, Y. Y., Kay-Lambkin, F., \&Hutchesson, M. J. (2019). Are Psychological Distress and resilience Associated with Dietary Intake Among Australian University Students?. International journal of environmental research and public health, 16(21), 4099. https://doi.org/10.3390/ijerph16214099

- World Health Organization (2020a, August). WHO Coronavirus disease (COVID-19) dashboard.Retrieved August11, 2021, from https://covid19.who.int/

- World Health Organization (2020b, August). Coronavirus: Overview. Retrieved August 11, 2020, from https://www.who.int/health-topics/ coronavirus\#tab=tab_1

- World Health Organization (2020c, August). WHO Director-General's opening remarks at the media briefing on COVID-19 - 11 March 
2020. Retrieved August 11, 2020, from https://www.who.int/dg/ speeches/detail/who-director-general-s-opening-remarks-at-the-mediabriefing-on-covid-19---11-march-2020

- Ying, Y., Ruan, L., Kong, F., Zhu, B., Ji, Y. \& Lou, Z. (2020). Mental health status among family members of health care workers in Ningbo, China, during the coronavirus disease 2019 (COVID-19) outbreak: a cross-sectional study. BMC Psychiatry, 20 (1), pp. 379.

- Yu, F., Cavadino, A., Mackay, L., Ward, K., King, A. \& Smith, M. (2020). Physical activity and personal factors associated with nurse resilience in intensive care units. J ClinNurs, 29: 32463262. https://doi.org/10.1111/jocn. 15338

- Zhang, Y. \& Ma, Z.F. Impact of the COVID-19 Pandemic on Mental Health and Quality of Life among Local Residents in Liaoning Province, China: A Cross-Sectional Study. (2020). Int. J. Environ. Res. Public Health, 17, 2381. 